\title{
Polynomial Law: A Better Alternative to Binary Exponential Backoff
}

\author{
Luca Barletta and Flaminio Borgonovo \\ Dipartimento di Elettronica, Informazione e Bioingegneria \\ Politecnico di Milano \\ I-20133 Milan, Italy \\ \{luca.barletta, flaminio.borgonovo\}@polimi.it
}

\begin{abstract}
In this paper we provide an approximate analysis that synthesizes results on the throughput and the delay of $S$ Aloha under a variety of backoff laws with an unlimited number of backoff stages. Past results, common to those about IEEE 802.11 protocols, show that, with the exponential backoff, the access-delay distribution is heavy tailed, and that, in order to alleviate the delay, throughput and number of users must be sacrificed. This paper uses an approximated and simple model recently introduced to produce results that are usually derived in a more complex framework. Here the model is extended to general backoff laws, including polynomial laws of type $\left(a+i^{\nu}\right)^{-1}$. We find that, when considering all performance factors, i.e., throughput, variance, access flexibility, and maximum number of users allowed, the polynomial law is to be preferred, with a degree $\nu$ that increases with the number of users that can be accommodated.
\end{abstract}

Index Terms-Slotted-Aloha, Backoff, Throughput, Delay, Unfairness, Decoupling Assumption, Random Access.

\section{INTRODUCTION}

In this paper we provide an approximate analysis that synthesizes results on the throughput and the delay of S-Aloha [1], [2] under a variety of backoff laws with an unlimited number of backoff stages. The goal is to get insights into a backoff scheme able to conjugate high throughput and bounded delay variance, even when the number of users $N$ is unknown and possibly very large, as it can be the case in future applications.

Starting with [3], the analysis of backoff schemes has been undertaken mainly with respect to IEEE 802.11, which, in fact, uses some form of S-Aloha embedded in its random access scheme, together with the Binary Exponential Backoff (BEB). In BEB the transmission slot of a packet that has suffered $i$ collisions, the backoff index, is taken as a uniform variable in a window of length $2^{i+i_{0}}$, where $i_{0}$ determines the initial length $W_{0}=2^{i_{0}}$. Many studies (a detailed reference list can be found, for example in [4]) have appeared on the throughput performance of this protocol with respect to its parameter settings. Results show that BEB can provide some relevant throughput even when the number of users goes to infinity. This is possible because the Exponential Backoff (EB) deals with the congestion by pushing a large part of users toward large backoff stages. Such effect, in turn, causes very large delays, resulting in an access-delay probability distribution that presents a heavy-tail behavior, to the point that, in many cases, the delay variance does not exist. To face this problem many authors have suggested new backoff schemes (see [5] for a comprehensive reference list), although the delay problem does appear still unsolved.

Due to the complexity of a thorough analysis, most of the works cited above use reduced and approximated mathematical models, many of them considering the saturation model, first introduced in [3], and based on the assumption that queues are always full, such that as soon as a packet is successfully transmitted at a station, immediately a new one is available for transmission. This model is somewhat simpler and pessimistic with respect to the one with real queues, and has been adopted in the hope that it presented a stable behavior and positive throughput, thus guaranteeing the stable behavior and the throughput of the more realistic one.

A further simplifying assumption, introduced in [3] and known as the decoupling assumption, has been largely used [6]-[9]. This assumption considers the behavior of different transmitters to be stationary in time and independent from station to station. Using both the above assumptions leads to a mean value analysis (MVA) and a fixed point equation [3], that provides in a simple way the (approximated) basic performance figures of the protocol.

Only a few authors have used the above models with respect to S-Aloha. In other cases throughput results are provided with respect to IEEE 802.11, so that their values strongly depends on the choice of the specific parameters of this protocol, such as the packet transmission time, the RTS-CTS time, the slot time and so on, making it difficult to extract information about the underlying S-Aloha. Furthermore, the elaborate passages required by the MVA, and the lack of close-from results, often obscure the role of parameters.

Recently, an analysis of EB for S-Aloha has appeared in [10] where the results for the decoupling assumption have been compared with more realistic ones, attained by the SemiPoisson Model (SPM), based on a reduced Markov Chain. It is shown that, although the decoupling assumption provides good throughput results for large values of the initial backoff window $W_{0}$, and a large number of users, it behaves fairly worse when considering a small $W_{0}$, as it is more suitable to S-Aloha. In fact, the initial window $W_{0}$ determines the bandwidth a user can grab when alone on the channel. The larger $W_{0}$ is, the smaller is the bandwidth it can use. The same is true for the access delay.

Unfortunately, the Markov Chain of SPM is numerically 
solvable for some specific parameters setting, such as those found in commercial applications, being its complexity too high in other cases, as some relevant to this paper. In these same cases, simulation does not help, since the convergence times are very long. The decoupling assumption presently remains, then, the only available tool able to catch the behavior of the throughput when the system parameters change.

We, therefore, in our investigations on the best parameters setting and the best backoff law, resort to the decoupling assumption model, knowing that our results can be mere indicative of an optimality, rather than providing exact quantitative results. More precisely, we adopt the Poisson Model (PM) introduced in [10], [11], which has been shown to be equivalent to decoupling assumption, but appears to be much simpler. We generalize this model to enclose any backoff law with increasing backoff window. To this respect, we prove some general properties about throughput that have already appeared, in part, in [5], although with a different technique. Our PM approach provides a simpler analysis and results when compared to those in [5], yielding in some cases close-form results.

In the access-delay analysis we adopt the approach in [11], [12], introducing additional theorems that allow to derive the tail of the probability distribution. We show conditions for the existence of delay moments of any order, generalizing those on the delay variance derived in [5]. These tools are used to investigate the optimal parameters setting and the backoff law to be preferred among EB and polynomial (POL) schemes, taking into account constraints on the delay variance and the flexibility of the protocol, i.e., the ability of providing a good balanced bandwidth on the entire range of $N$, from 2 up to a maximum number of users.

Our results indicate that POL is a good candidate law, able to provide throughput performance comparable or better than EB, and with more favorable delay distribution and flexibility. Similar results have been noted in [5], where a polynomial degree equal to 2 is suggested. Here we find that the polynomial degree must be increased as the number of users to be served enlarges.

The paper is organized as follows. In Sec. II we set the system parameters and derive some basic relations and results about the model. Section III is devoted to the Poisson model, and provide throughput numerical results. In Sec. IV we characterize the access-delay distribution results, while in Sec. V we evaluate the delay variance and provide numerical results about the maximum number of users $N_{\max }$ that can be accommodated under throughput and access-delay variance constraints. The validation of theoretical results is provided by simulation in Sec. VI. Conclusions are given in Sec. VII.

\section{PREliminary Results}

In this paper we investigate general backoff laws, where at each time a transmission occurs with probability $\beta_{i}$. Numerical results are attained for the following EB law

$$
\beta_{i}=b^{-i-i_{0}},
$$

and the POL law

$$
\beta_{i}=\left(a+i^{\nu}\right)^{-1}
$$

The above laws are of the memoryless type, slightly different from the Window type as considered, for BEB, in the IEEE 801.11. However, we can prove that, when used with the decoupling assumption, they provide exactly the same results of the window type. The reason is that, decoupling assumption only deals with mean values, which can be made equal in the two cases.

In [13] it has been proven that the exponential law (1) provides ergodic behavior and fair throughput for $b>1$ and $i_{0}>1$. The very same arguments can be used to show that the same holds for law (2) with $a>1$ and $\nu>1$.

We note that, when changing parameter $i_{0}$, or $b$, in (1), or parameter $a$ in (2), we change the bandwidth that is taken when only a single user is present, that is equal to $b^{-i_{0}}$ and $1 / a$ in the two cases, and that we dub protocol flexibility. Since the appealing feature of Aloha is the ability to provide, more or less, the same bandwidth whichever the number of users is, in the following we compare the protocols and their different settings by fixing the flexibility parameter. Since the case $i_{0}=1$ is the boundary for stable operation of EB, we safely assume $i_{0} \geq 2$. On the other side, it is well known, as we show below, that with $b=2$ the protocol is overreacting, i.e., users refrain too much from transmission when the backoff index increases. As a consequence the traffic on the channel is chocked, and the throughput is less than what can be obtained with smaller $b$ values. It has also been shown in [11] that increasing $b$ increases the delay and makes the tail of the delay distribution more unfavorable. Therefore, we will not consider values of $b$ greater than 2 . This leads to a reasonable flexibility parameter equal to $1 / 4$.

The system is described by a Markov chain whose Markovian state is $\left(N_{0}, N_{1}, \ldots, N_{i}, \ldots\right)$, where $N_{i}$ represents the number of users with backoff index $i$, whose expectation is denoted by $n_{i}$. The solution of the chain provides the joint distribution of the above variables, from which all the performance figures can be derived. Unfortunately, this is a too complex task to be attempted in a general setting. In [11], the complexity of the above chain is reduced by means of some approximations, so that in some cases, results can be derived with a precision that is increasing with complexity. Unfortunately, this approximation, which provides practically exact results for $b=2$, does not work well with smaller values of $b$, and with the POL model.

On the other side, the Poisson model introduced in [10], [11], does provide approximate results, even in the order of $10 \%$ in some cases. However, the behavior of the model, with respect to parameter changes, well reflects the behavior of the real system, and therefore can be usefully used to attain good insights and suggestions for optimal choices.

\section{A. General Setting}

The whole system can be conveniently represented by a network of stages, as shown in Fig. 1, where $\lambda_{i}$, the overall transmission rate at stage $i$, and $s_{i}$, the throughput of stage $i$, represent respectively the rates of user flows into and out stage $i$. 


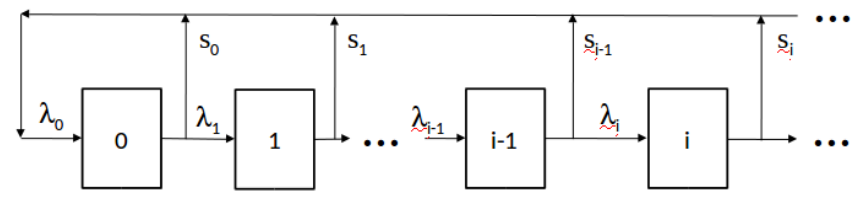

Figure 1. Scheme of the backoff mechanism as a network of stages.

The throughput of stage $i$ can be expressed as

$$
s_{i}=\mathrm{E}\left[\gamma_{i}\left(1, N_{i}\right) \prod_{k=0, k \neq i}^{\infty} \gamma_{k}\left(0, N_{k}\right)\right],
$$

where the average is taken over the joint distribution of the $N_{i}$ 's, and

$$
\gamma_{i}\left(k, N_{i}\right)=\left(\begin{array}{c}
N_{i} \\
k
\end{array}\right)\left(\beta_{i}\right)^{k}\left(1-\beta_{i}\right)^{N_{i}-k}
$$

is the probability of having $k$ transmissions in stage $i$. The throughput is evaluated as

$$
\lambda_{0}=\sum_{i=0}^{\infty} s_{i} .
$$

The "routing" probability out of stage $i$, defined as $\alpha_{i}=$ $\frac{\lambda_{i+1}}{\lambda_{i}}$, is given by:

$$
1-\alpha_{i}=\frac{s_{i}}{\lambda_{i}}=\frac{s_{i}}{n_{i}} t_{i}
$$

where the last passage comes from Little's result [14], being $t_{i}$ the average time the user spends in stage $i$, and equal to $\beta_{i}^{-1}$. We also have

$$
\lambda_{i}=\lambda_{0} \alpha_{0} \alpha_{1} \ldots \alpha_{i-1}, \quad i \geq 1,
$$

and again using Little's result, we can express $N$ as

$$
N=\sum_{i=0}^{\infty} n_{i}=\sum_{i=0}^{\infty} \lambda_{i} t_{i}=\sum_{i=0}^{\infty} \lambda_{i} \beta_{i}^{-1} .
$$

In the following we make use of the Poisson model, introduced in [10], that we generalize below.

\section{POISSON MODEL}

The Poisson model assumes that the stationary distribution of $\left(N_{0}, N_{1}, \ldots, N_{i}, \ldots\right)$ is a joint Poisson distribution, independent from stage to stage. Since a user with index $i$ transmits independently of others with probability $\beta_{i}$, the distribution of transmitting users $N_{i}$ at stage $i$ is still Poisson, independent of $N_{j}, j \neq i$, with average $\lambda_{i}=n_{i} \beta_{i}$. Since the sum of independent Poisson variables is still Poisson distributed, the traffic on the channel is as such, with average $\Lambda=\sum_{k=0}^{\infty} \lambda_{k}$. We then have

$$
\begin{gathered}
\alpha_{i}=1-e^{-\Lambda}, \\
\lambda_{0}=\Lambda e^{-\Lambda},
\end{gathered}
$$

and, by (5), (6), (7) and (8), being $\lambda_{i}=\lambda_{0} \alpha^{i}$, we get

$$
N=\Lambda e^{-\Lambda} \sum_{i=0}^{\infty}\left(1-e^{-\Lambda}\right)^{i} / \beta_{i} .
$$

We note that the above summation must converge since the system is known to work in stationary conditions and $N$ is a given finite number.

In the two considered backoff cases, equation (9) has a close-form expression that is derived next. By inspecting those expressions, we can state

Property 1. The RHS of (9) is a monotonically increasing function of $N$.

Therefore, equation (9) can be solved to get a unique $\Lambda$, given $N$, and all other metrics. Furthermore we have:

Property 2. For any given parameters setting, if $N$ increases, then traffic $\Lambda$ increases. The maximum channel traffic $\Lambda^{*}$ is reached for $N=\infty$.

Property 3. The maximum throughput in (8) is $e^{-1}$ and is attained in $\Lambda=1$.

However, in order the maximum throughput to be reached, the protocol parameters must be tuned, if possible, in order that we get $\Lambda=1$. The maximum channel traffic $\Lambda^{*}$, reached when $N=\infty$, is attained when (9) diverges in $\Lambda=\Lambda^{*}$, while converging for $\Lambda<\Lambda^{*}$. Using the ratio test for the convergence, we can easily prove the following

Property 4. A finite maximum channel traffic, $\Lambda^{*}<\infty$, is reached only if

$$
\lim _{i \rightarrow \infty} \frac{\beta_{i+1}}{\beta_{i}}=1-e^{-\Lambda^{*}}
$$

and the system provides positive throughput. Otherwise, if

$$
\lim _{i \rightarrow \infty} \frac{\beta_{i+1}}{\beta_{i}} \geq 1
$$

(9) diverges when $\Lambda^{*}=\infty$, and the throughput is zero.

Therefore, in order to reach the maximum throughput in $\Lambda^{*}=1$ with $N=\infty$, we must have

$$
\lim _{i \rightarrow \infty} \frac{\beta_{i+1}}{\beta_{i}}=1-e^{-1} \text {. }
$$

To end this part, we must note that many of the result and properties above have already been derived in equivalent form in [5], although in a quite more complex context that uses the decoupling assumption.

\section{A. EB case}

Using (1), we get immediately

$$
N=b^{i_{0}} \frac{\Lambda e^{-\Lambda}}{1-b\left(1-e^{-\Lambda}\right)} .
$$

We also get

$$
\begin{gathered}
\Lambda<\ln \frac{b}{b-1}=\Lambda^{*} \\
\lambda_{0}<\frac{b-1}{b} \ln \frac{b}{b-1} .
\end{gathered}
$$

The model works up to $\Lambda=\Lambda^{*}=\ln \frac{b}{b-1}$, approximately equal to 0.7 for $b=2$, where the throughput is $\lambda_{0}=\ln (2) / 2 \approx 0.34$. This clearly shows that BEB is 
unable to provide maximum throughput. The throughput of this model reaches maximum when $\ln \frac{b}{b-1}=1$, which implies $b=\frac{1}{1-e^{-1}} \approx 1.58$, according to Proposition 4, regardless of $i_{0}$. These latter results were already derived in [6] with the decoupling assumption, again in a more complex framework.

From inspection of (10) we further have:

Property 5. For given $N$ and $i_{0}$, if we increase b, then traffic $\Lambda$ decreases.

Property 6. For given $b$ and $\Lambda$, if we increase $i_{0}$, then $N$ is increased, and the throughput curve $\lambda_{0}(N)$ is merely shifted.

\section{B. POL case}

Here we have

$$
N=\Lambda\left(a+e^{-\Lambda} \sum_{i=0}^{\infty}\left(1-e^{-\Lambda}\right)^{i} i^{\nu}\right),
$$

which, for example, for $\nu=1,2,3,4$, provides the closed forms:

$$
\begin{gathered}
N=\Lambda\left(a+\frac{u(\Lambda)}{e^{-\Lambda}}\right), \\
N=\Lambda\left(a+\frac{u(\Lambda)^{2}+u(\Lambda)}{e^{-2 \Lambda}}\right), \\
N=\Lambda\left(a+\frac{u(\Lambda)^{3}+4 u(\Lambda)^{2}+u(\Lambda)}{e^{-3 \Lambda}}\right), \\
N=\Lambda\left(a+\frac{u(\Lambda)^{4}+11 u(\Lambda)^{3}+11 u(\Lambda)^{2}+u(\Lambda)}{e^{-4 \Lambda}}\right),
\end{gathered}
$$

where $u(\Lambda)=1-e^{-\Lambda}$. Since with this law it is

$$
\lim _{i \rightarrow \infty} \frac{\beta_{i+1}}{\beta_{i}}=1
$$

POL can not provide positive throughput (9) as $N$ diverges. Furthermore we have

Property 7. If we increase the flexibility parameter a by step $\Delta a$, then $N$ is increased by $\Lambda \Delta a$, the throughput curve $\lambda_{0}(N)$ is shifted to the right and scaled with $N$.

\section{Throughput with Constant Flexibility}

With the PM, we have derived the throughput curves for EB (10) and POL (12) shown respectively in Fig. 2 and 3 for different values of the parameters, keeping the flexibility $b^{-i_{0}}$ and $1 / a$ at the constant value 0.25 .

The curve for $b=1.6$ is practically the one that reaches throughput $e^{-1}$ when $N=\infty$, as shown in Sec. III-A. For $b<$ 1.6 the asymptotic throughput decreases while the maximum is reached in $N=6,5,4$ for respectively $b=1.2,1.1,1.05$.

On the other side, the POL case in Fig. 3 shows a throughput that asymptotically decreases to zero, as shown in Sec. III-B. Therefore, such a law can be considered only when the maximum value of $N$ is bounded. For example, we see that $\nu=4$ provides good throughput up to at least $N=10000$.

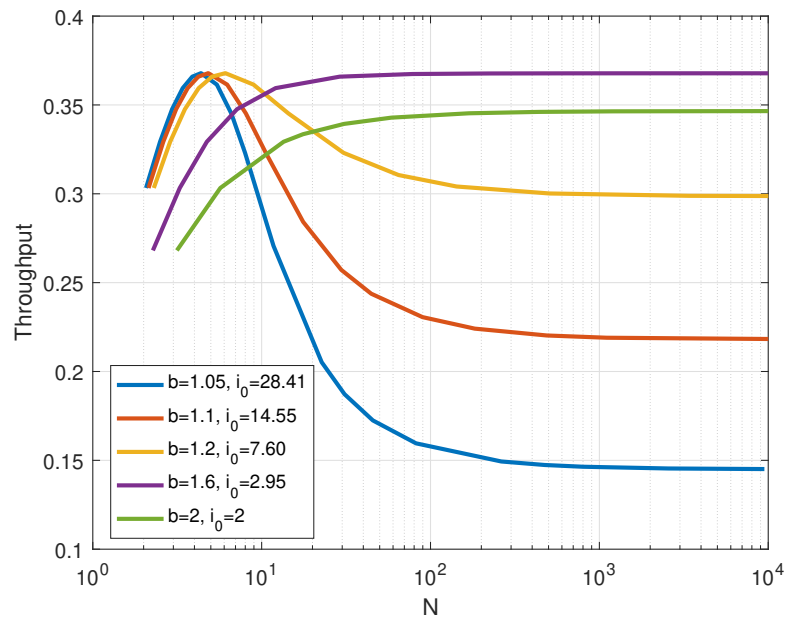

Figure 2. Throughput versus the number of users for EB and different parameters value with constant flexibility $b^{-i_{0}}=0.25$.

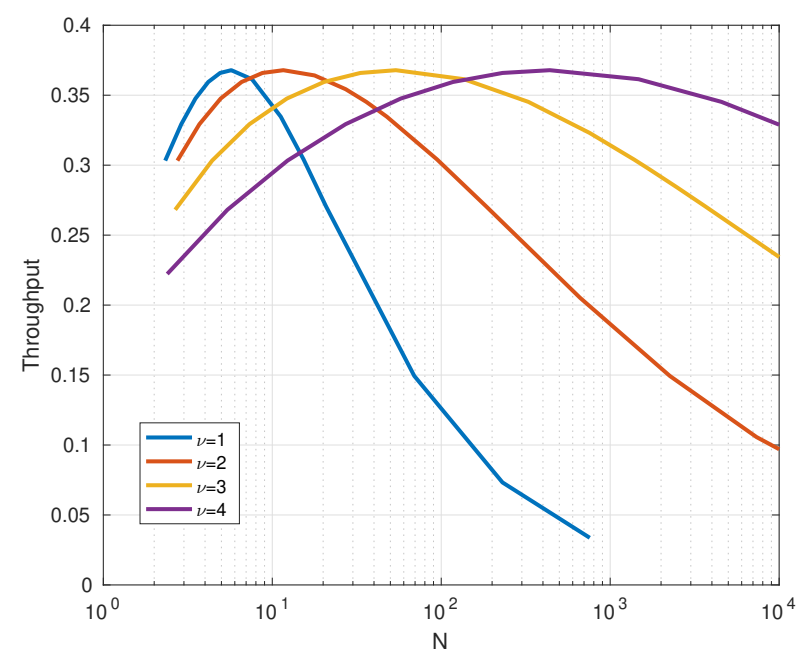

Figure 3. Throughput versus the number of users for POL and different parameter value with constant flexibility $1 / a=0.25$.

We must note that the advantage of EB in providing positive throughput even for an infinite number of users is only apparent since, as it is proved in the next section, a large part of them is pushed toward high index stages in such a way that the delay experienced can be hardly considered for practical purposes.

To end this section, it is worth mentioning the lack of accuracy of throughput values at low $N$, especially for $N=1$, where all curves are expected to converge to 0.25 , and more so with $b^{-i_{0}}=2$, where in $N=1$ the throughput is 0.5. However, as explained in Sec. I this lack of accuracy represents, for the moment, the price to be paid to get an overall insight of the protocol performance.

\section{ACCESS-DELAY}

The access delay is represented by the random variable (RV)

$$
D_{R}=\sum_{k=0}^{R} T_{k}
$$


where $T_{k}$ is the geometric RV with average $1 / \beta_{k}$, that represents the time spent in stage $k$ by a user, and $R$ is the random index of the stage where the transmission is successful.

The throughput $\lambda_{0}$, evaluated as described in Sec. III, immediately provides the first-order delay moment as

$$
d_{R} \triangleq \mathrm{E}\left[D_{R}\right]=\frac{N}{\lambda_{0}} \text {. }
$$

As simple as it is, relation (18) has been overlooked in many of the works appeared in the literature. It is readily proved by Little's Result and by observing that the throughput of each user is $\lambda_{0} / N$. On the other side, with the saturation model, the average number of packets in the transmission buffer is always one. This result shows that the setting that provides the highest throughput also gives the smallest average access delay.

The complementary cumulative distribution function (CCDF) of $D_{R}$ can be expressed as

$$
P\left(D_{R}>d\right)=\sum_{r=0}^{\infty} P(R=r) P\left(D_{r}>d\right),
$$

where the probability mass function of $R$, by (4), (5) and (7), is

$$
P(R=i)=\alpha^{i}(1-\alpha), \quad i=0,1, \cdots .
$$

Conditioned to $R=r$, the probability mass function of $D_{r}$ has average

$$
d_{r}=\mathrm{E}\left[D_{r}\right]=\sum_{k=0}^{r} 1 / \beta_{k}
$$

In the Appendix we prove the following

Theorem 1. The random variable $D_{r} / d_{r}$ converges in distribution, for $r \rightarrow \infty$, to a random variable $\Delta$ with pdf

$$
p_{\Delta}(x)=\sum_{k=0}^{\infty} \mu_{k} e^{-\mu_{k} x} \prod_{j \neq k, j=0}^{\infty} \frac{\mu_{j}}{\mu_{j}-\mu_{k}}, \quad x>0,
$$

where $\mu_{k}^{-1}=\lim _{r \rightarrow \infty}\left(\beta_{r-k} d_{r}\right)^{-1}$ for $k=0,1, \ldots$, provided that the limits exist.

We now prove the following:

Theorem 2. For large values of $i$ we have

$$
P\left(D_{R}>d_{i}\right) \approx A \alpha^{i} .
$$

Proof: Given $r_{1}$ we have

$$
\lim _{d \rightarrow \infty} P\left(D_{r_{1}}>d\right)=0,
$$

which means that given any $\varepsilon>0$ we can always find $d^{\prime}\left(r_{1}, \varepsilon\right)$ such that for $d>d^{\prime}$

$$
\sum_{r=0}^{r_{1}-1} P(R=r) P\left(D_{r}>d\right) \leq \varepsilon .
$$

Also, given $d$ we have

$$
\lim _{r \rightarrow \infty} P\left(D_{r}>d\right)=1
$$

which shows that given any $\varepsilon^{\prime}>0$ we can always find $r_{2}\left(d, \varepsilon^{\prime}\right)$ such that for $r>r_{2}$

$$
\begin{gathered}
\sum_{r=r_{2}+1}^{\infty} P(R=r)-\varepsilon^{\prime} \leq \\
\sum_{r=r_{2}+1}^{\infty} P(R=r) P\left(D_{r}>d\right) \leq \sum_{r=r_{2}+1}^{\infty} P(R=r) .
\end{gathered}
$$

Since $\varepsilon$ and $\varepsilon^{\prime}$ can be made as small as wanted, for $d>$ $d^{\prime}\left(r_{1}, \varepsilon\right)$ and for a suitable $r_{2}$ we can write

$P\left(D_{R}>d\right) \approx \sum_{r=r_{1}}^{r_{2}} P(R=r) P\left(D_{r}>d\right)+\sum_{r=r_{2}+1}^{\infty} P(R=r)$.

Then, by Theorem 1, we can always find an integer $r_{1}$ so that delay (19) can be written as

$$
P\left(D_{R}>d\right) \approx \sum_{r=r_{1}}^{r_{2}} P(R=r) P\left(\Delta>\frac{d}{d_{r}}\right)+P\left(R>r_{2}\right),
$$

for $d \geq d^{\prime}\left(r_{1}\right)$, where the approximation becomes equality in the limit $d \rightarrow \infty$. For $d=d_{i}$, by (26), we have

$P\left(D_{R}>d_{i}\right) \approx \sum_{r=i-a_{1}}^{i+a_{2}} P(R=r) P\left(\Delta>\frac{d_{i}}{d_{r}}\right)+P\left(R>r_{2}\right)$,

having denoted $r_{1}=i-a_{1}$ and $r_{2}=i+a_{2}$. For large values of $r_{1}, r_{2}$ and $i, r_{1}<i<r_{2}$, using (20) we get (23) where

$$
A=(1-\alpha) \sum_{k=-a_{1}}^{a_{2}} \alpha^{k} P\left(\Delta>\frac{d_{i}}{d_{i+k}}\right)
$$

and $P\left(R>r_{2}\right)$ has been disregarded.

\section{A. EB case}

Here we have

$$
d_{i}=\sum_{k=0}^{i} b^{i_{0}+k}=b^{i_{0}} \frac{b^{i+1}-1}{b-1} \approx h b^{i},
$$

where the approximation holds for large $i$. Defining

$$
\alpha=b^{-\zeta(N)},
$$

and using (23) and (26) provides

$$
P\left(D_{R}>d_{i}\right) \approx A d_{i}^{-\zeta},
$$

or

$$
P\left(D_{R}>d\right) \approx A d^{-\zeta}
$$

By the definitions we can write

$$
\zeta=-\frac{\ln \alpha}{\ln b}=-\frac{\ln \left(1-e^{-\Lambda}\right)}{\ln b}
$$

which shows that the slope $\zeta$ decreases as $N$ increases since, by Property 2, also $\Lambda$ increases. With $N=\infty$, by Property 4 , we have $1-e^{-\Lambda^{*}}=1 / b$, which gives $\zeta=1$. Furthermore, $\zeta$ reaches its maximum value when $\Lambda$ is minimum, which happens for $N=2$. 
Property 8. The slope $\zeta$ of the delay CCDF decreases as $N$ increases reaching 1 with an infinite number of users.

Since delay moments are given by

$$
\begin{aligned}
\mathrm{E}\left[\left(D_{R}\right)^{k}\right] & =\int_{0}^{\infty} k d^{k-1} P\left(D_{R}>d\right) \mathrm{d} d \\
& \approx A \int_{0}^{\infty} k d^{-(\zeta-k+1)} \mathrm{d} d,
\end{aligned}
$$

they only exist if

$$
\zeta>k
$$

and the existence of moments is dictated by Property 8 .

B. POL case

Here we have

$$
d_{i}=\mathrm{E}\left[D_{i}\right]=\sum_{k=0}^{i} \frac{1}{a+k^{\nu}} \approx h^{\prime} i^{\nu},
$$

and

$$
i=h^{\prime \prime} d_{i}^{1 / \nu}
$$

which used into (23) provides

$$
P\left(D_{R}>d_{i}\right) \approx A \alpha^{h^{\prime \prime} d_{i}^{1 / \nu}}
$$

or

$$
P\left(D_{R}>d\right) \approx A \alpha^{h^{\prime \prime} d^{1 / \nu}} .
$$

As the tail of the distribution decreases exponentially, we have:

Property 9. With the polynomial law, and finite $N$, moments of any order exist, and their values increase with $\nu$.

\section{Delay-VARiance}

Since the value of the access-delay variance is a key parameter in designing a practical system, and since such variance increases with the number of users $N$, in this section we investigate, for a given flexibility parameter and throughput, the upper bound on $N$ as the delay variance changes. To this purpose we derive the expression of the second order moment as

$$
\begin{aligned}
\mathrm{E}\left[D_{R}^{2}\right] & =\sum_{r=0}^{\infty} \mathrm{E}\left[\left(D_{r}\right)^{2}\right] p_{R}(r) \\
& =\sum_{r=0}^{\infty}\left(\sum_{k=0}^{r} \frac{2-\beta_{k}}{\beta_{k}^{2}}+2 \sum_{i=0}^{r-1} \sum_{j=i+1}^{r} \frac{1}{\beta_{i} \beta_{j}}\right) \alpha^{r}(1-\alpha),
\end{aligned}
$$

where we have used the second order moment of the geometric RV $D_{r}$. This expression can be used to prove the following

Theorem 3. If

$$
\lim _{i \rightarrow \infty} \frac{\beta_{i+1}}{\beta_{i}}=1,
$$

then (37) exists for any $N$. Otherwise, if

$$
\lim _{i \rightarrow \infty} \frac{\beta_{i+1}}{\beta_{i}}<1,
$$

(37) exists for $N$ up to some finite limit.

The above theorem, and its proof, can be found in [5], with a suitable change of variables. It generalizes the results on second moments found in Sec. IV.

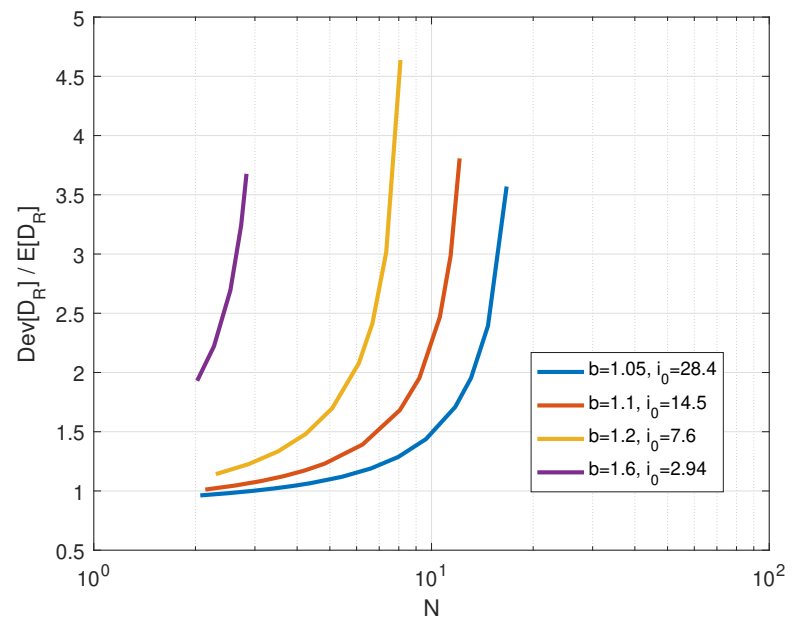

Figure 4. Normalized standard deviation of the access delay for EB as function of $N$ for different parameters values with constant flexibility $b^{-i_{0}}=0.25$.

\section{A. EB case}

From Theorem 3 we see that the second delay moment exists up to some $N_{\max }$. For the second moment of $D_{R}$ in few passages we get

$$
\begin{aligned}
\mathrm{E}\left[\left(D_{r}\right)^{2}\right] & =b^{i_{0}} \sum_{k=0}^{r}\left(2 b^{2 k+i_{0}}-b^{k}\right)+2 b^{2 i_{0}} \sum_{i=0}^{r-1} b^{i} \sum_{j=i+1}^{r} b^{j} \\
& =b^{i_{0}}\left(2 b^{i_{0}} \frac{b^{2(r+1)}-1}{b^{2}-1}-\frac{b^{r+1}-1}{b-1}\right) \\
& +\frac{2 b^{2 i_{0}}}{b-1}\left(b^{r+1} \frac{b^{r}-1}{b-1}-b \frac{b^{2 r}-1}{b^{2}-1}\right) .
\end{aligned}
$$

Using (38) in (37), we see that if we want the second moment to exist we must require $b^{2} \alpha=b^{2}\left(1-e^{-\Lambda}\right)<1$ or, equivalently, $\Lambda<\ln \left(b^{2} /\left(b^{2}-1\right)\right)$. By (10) the latter can be used to get $N_{\max }$, which represent the asymptotes of the curves in Fig. 4 (the case $b=2$ is not reported being $N_{\max }=1.73$ ). Under this constraint, the second moment of $D_{R}$ is

$$
\begin{aligned}
\mathrm{E}\left[\left(D_{R}\right)^{2}\right] & =b^{i_{0}}\left(2 b^{i_{0}} \frac{b^{2} \mathrm{E}\left[b^{2 R}\right]-1}{b^{2}-1}-\frac{b \mathrm{E}\left[b^{R}\right]-1}{b-1}\right) \\
& +\frac{2 b^{2 i_{0}}}{b-1}\left(b \frac{\mathrm{E}\left[b^{2 R}\right]-\mathrm{E}\left[b^{R}\right]}{b-1}-b \frac{\mathrm{E}\left[b^{2 R}\right]-1}{b^{2}-1}\right) \\
& =b^{i_{0}} \frac{2 b^{i_{0}}-1+\alpha b^{2}}{\left(1-\alpha b^{2}\right)(1-\alpha b)} .
\end{aligned}
$$

The expected value of $D_{R}$ is, by (18)

$$
\mathrm{E}\left[D_{R}\right]=b^{i_{0}} \frac{1}{1-\alpha b},
$$

and the variance is

$$
\operatorname{Var}\left[D_{R}\right]=b^{i_{0}} \frac{2 b^{i_{0}}-1+\alpha b^{2}}{\left(1-\alpha b^{2}\right)(1-\alpha b)}-b^{2 i_{0}} \frac{1}{(1-\alpha b)^{2}} .
$$

In Fig. 4 we show the standard deviation normalized to the mean, $\operatorname{Dev}\left[D_{R}\right] / \mathrm{E}\left[D_{R}\right]$, of the access delay, versus $N$, for different parameters values with the same access flexibility $b^{-i_{0}}=0.25$. This metrics do not exist for $i_{0}=2, b=2$, 


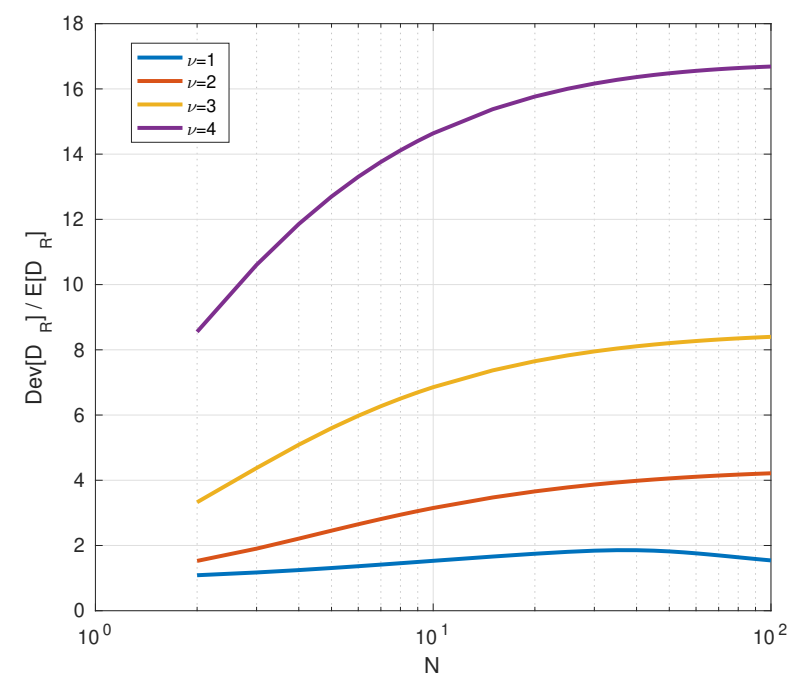

Figure 5. Normalized standard deviation of the access delay for POL versus $N$ for different parameters values with constant flexibility $1 / a=0.25$.

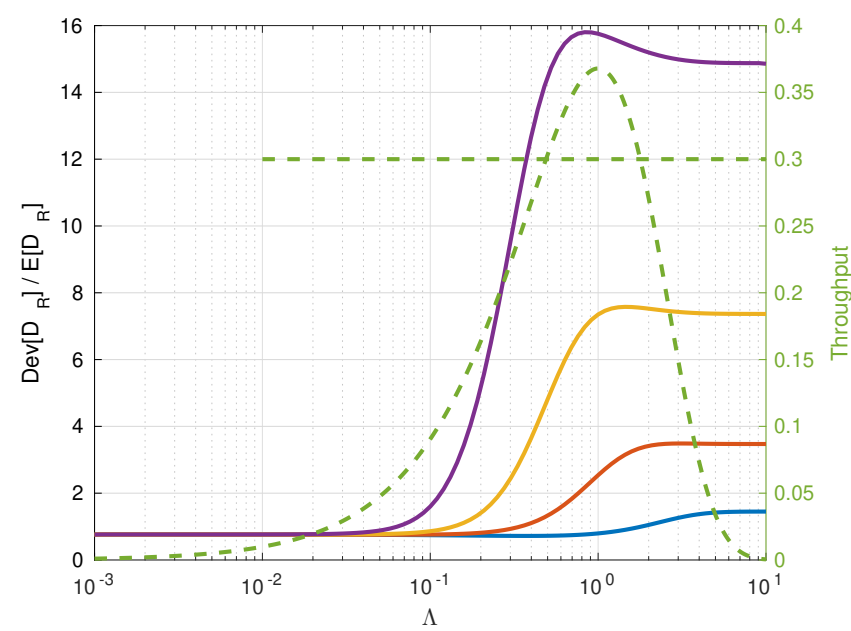

Figure 6. Normalized standard deviation of the access delay for POL versus the channel traffic $\Lambda$ for different parameters values with constant flexibility $1 / a=0.25$. The throughput curve is also reported as a dashed line and refers to the axis shown on the right.

then exists and improves for larger $i_{0}$. In the best case, $b=$ $1.05, i_{0}=28.4$, we have $N_{\max }=18$, which shows that EB is not practicable when $N$ is beyond this point.

\section{B. POL case}

Here Theorem 3 shows that the second delay moment always exists. Unfortunately, we can not get a close-form expression and, therefore, we resorted to numerical evaluations of (37).

In Fig. 5 we show the normalized standard deviation (NSD), as in Fig. 4, for the POL case and the same access flexibility $1 / a=0.25$ as with EB. Here the variance is much more favorable than with $\mathrm{EB}$ although the ratio $\operatorname{Dev}\left[D_{R}\right] / \mathrm{E}\left[D_{R}\right]$ greatly increases with $\nu$.

In Fig. 6 we show the NSD as in Fig 5, plotted against $\Lambda$. This figure also reports the throughput curve $\Lambda e^{-\Lambda}$, which

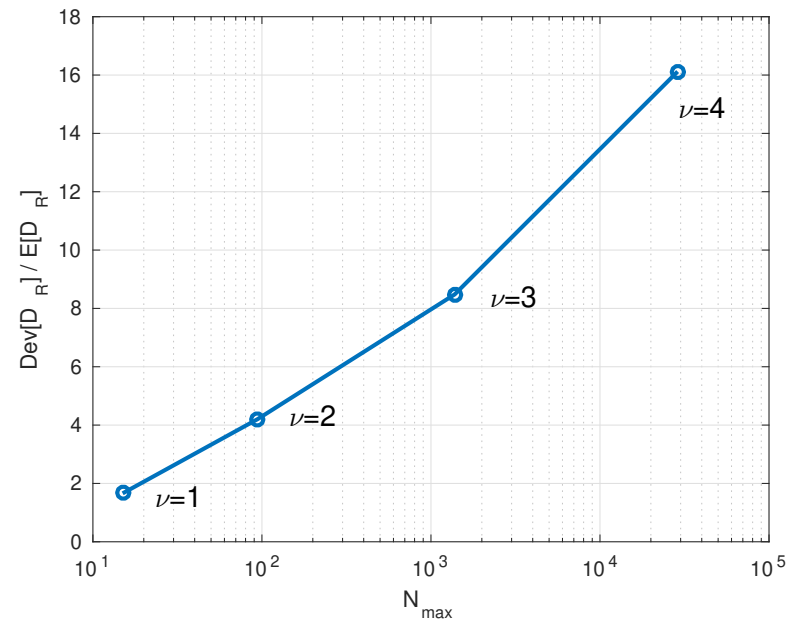

Figure 7. Normalized standard deviation of the access delay for POL versus the maximum number of users $N_{\max }$ that can be accommodated with a throughput no less than 0.3 , and with constant flexibility $1 / a=0.25$. The corresponding polynomial degree, from 1 to 4 , is also reported at the different points of the curve.

is used to evaluate Fig. 7. The latter figure provides the maximum NSD that is achieved while accommodating up to $N_{\text {max }}$ users with the requirement of a minimum throughput equal to 0.3 , and flexibility equal to 0.25 . To do so, we consider the rightmost point at which the horizontal line in Fig. 6, that corresponds to throughput equal to 0.3 , crosses the throughput curve. A vertical line from this point crosses the NSD curves at four points, that we have reported in Fig. 7 versus $N$, as obtained by (10). We can not provide a similar curve for EB since Fig. 4 shows that EB has no variance for $N>18$.

From the results of this section we can conclude that, taking into account the delay as expressed by Fig. 7, the POL backoff is to be preferred to EB. In any cases, we must pay for a larger number of users with a great increase in the delay variance, since a higher degree of the polynomial backoff is required.

\section{VALIDATION}

The results so far exposed, show that the POL with $\nu=2$ is a good candidate for a system that can accommodate up to 100 users. However, in order to definitely assess this conclusion, we need to validate the analytical results derived with the Poisson model. In [10], [11] we have derived practically exact throughput and delay values for the BEB case, i.e., EB with $b=2, i_{0}=2$, and validation is attained by comparing those results with the ones in Fig. 2. In Fig. 8 we compare, for $\nu=2$, the theoretical throughput and the standard deviation that we have already seen in Fig. 3 and 5, with those attained by simulation. Similar results hold with different parameters. As expected, discrepancies are present, especially for low values of $N$; however, the overall error and behavior of the model are such that the conclusions of the analysis are confirmed.

Since the POL backoff with $\nu=2$ presents a less reactive probability law with respect to $\mathrm{EB}$ with $b=2, i_{0}=2$, one might suspect that the POL mechanism is far less prompt than EB in following changes in $N$. To investigate such a dynamic 


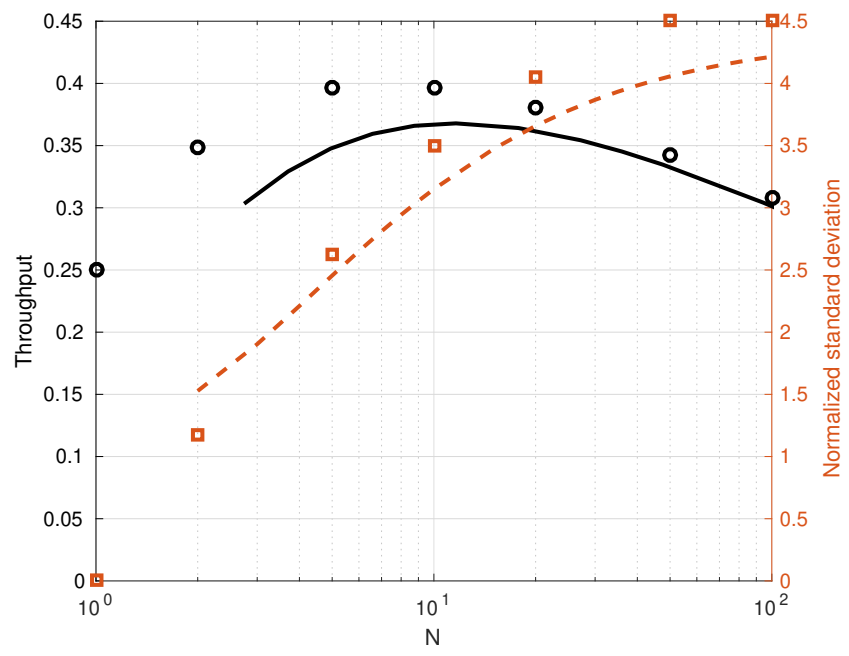

Figure 8. Throughput and normalized standard deviation of the access delay for POL with $\nu=2$ and $1 / a=0.25$ versus of the number of users $N$. Simulation results are reported as dots.

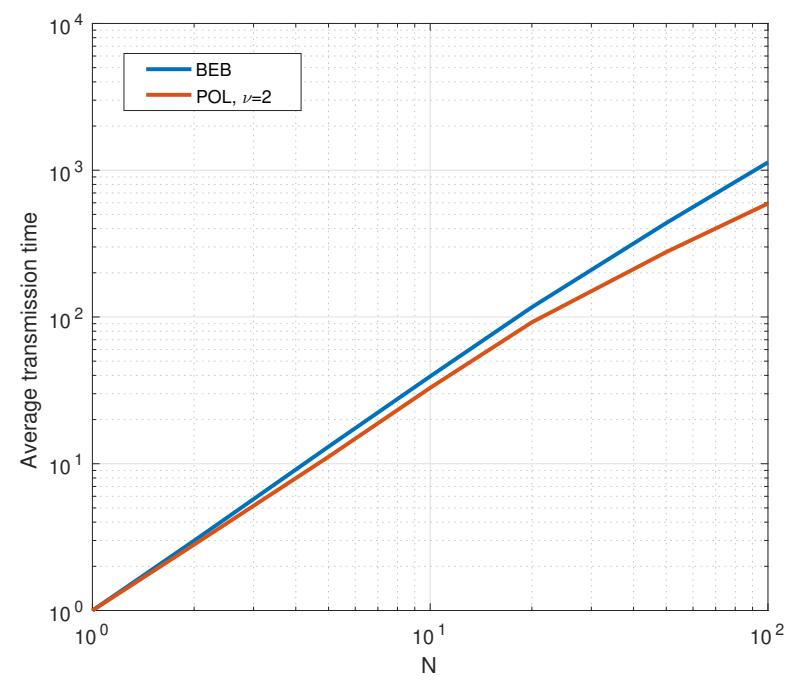

Figure 9. Average time to the complete transmission of $N$ packets starting with zero indexes using BEB and POL with $\nu=2$ and flexibility 0.25 .

behavior, we have considered the average time it takes to $N$ users to correctly transmit just one packet each, starting at time zero with zeros backoff indexes. In Fig. 9 we have reported the measure of such a time attained by simulation, using BEB and POL. As we can see, the suspect has no ground since, even here, POL performs slightly better than EB.

\section{CONCLUSIONS}

In this paper we have produced an analytical and numerical investigation that synthesizes throughput and delay results over a wide range of backoff laws. Our analytical investigations have used an approximated model that produces in a simple way many properties concerning some general backoff laws, some of them already derived in the literature in a more complex framework. Numerical investigations has aimed at determine optimal settings and optimal backoff laws, that can produce a finite variance for the access delay, still providing a comparable bandwidth over an entire range for the number of users. Our results indicate that the polynomial backoff law is by far preferable with respect to the traditional exponential law, and, if no more than 100 users are considered, a good candidate law is the polynomial law of second degree.

\section{APPENDIX}

\section{PROOF OF TH. 1}

For large $r$, it is standard to show that the scaled Geometric random variable $T_{r} / \mathrm{E}\left[D_{r}\right]=T_{r} / d_{r}$ converges in distribution to an Exponential random variable with average $\mu_{0}^{-1}=\lim _{r \rightarrow \infty}\left(\beta_{r} d_{r}\right)^{-1}$, provided that $\mu_{0}^{-1}<\infty$. Pretty much in the same way, one can proceed backwards to show that $T_{r-k} / \mathrm{E}\left[D_{r}\right]$ converges in distribution to an Exponential random variable with average $\mu_{k}^{-1}=\lim _{r \rightarrow \infty}\left(\beta_{r-k} d_{r}\right)^{-1}$, for any finite $k=1,2, \cdots$. The sum of all random variables $T_{r-k} / d_{r}$ for $k=0,1, \ldots$ gives rise to the convolution of exponential random variables with different averages, whose result is (22) [15].

\section{REFERENCES}

[1] N. Abramson, "The Aloha system: Another alternative for computer communications," in Proc. Fall Joint Computer Conf., vol. 37, Nov. 1970, pp. 281-285.

[2] L. G. Roberts, "Aloha packet system with and without slots and capture," in ARPA Satellite System Note, no. 8, Jun. 1972.

[3] G. Bianchi, "Performance analysis of the IEEE 802.11 distributed coordination function," IEEE J. Select. Areas Commun., vol. 18, no. 3, pp. 535-547, March 2000.

[4] L. Dai and X. Sun, "A unified analysis of IEEE 802.11 DCF networks: Stability, throughput, and delay," IEEE Transactions on Mobile Computing, vol. 12, no. 8, pp. 1558-1572, Aug 2013.

[5] X. Sun and L. Dai, "Backoff design for IEEE 802.11 DCF networks: Fundamental tradeoff and design criterion," IEEE/ACM Transactions on Networking, vol. 23, no. 1, pp. 300-316, Feb 2015.

[6] B.-J. Kwak, N.-O. Song, and L. Miller, "Performance analysis of exponential backoff," IEEE/ACM Trans. Netw., vol. 13, pp. 343-355, April 2005.

[7] A. Kumar, E. Altman, D. Miorandi, and M. Goyal, "New insights from a fixed-point analysis of single cell IEEE 802.11 WLANs," IEEE/ACM Trans. Netw., vol. 15, no. 3, pp. 588-601, June 2007.

[8] J. Cho, J.-Y. Le Boudec, and Y. Jiang, "On the asymptotic validity of the decoupling assumption for analyzing 802.11 MAC protocol," IEEE Trans. Inf. Theory, vol. 58, no. 11, pp. 6879-6893, Nov 2012.

[9] C. Bordenave, D. McDonald, and A. Proutiere, "A particle system in interaction with a rapidly varying environment: Mean field limits and applications," arXiv:math/0701363v3, Nov 2013.

[10] L. Barletta, F. Borgonovo, and I. Filippini, "The S-Aloha capacity: Beyond the $e^{-1}$ myth," in IEEE INFOCOM 2016 - The 35th Annual IEEE International Conference on Computer Communications, April 2016, pp. 1-9.

[11] $\_$, "The throughput and access delay of slotted-Aloha with exponential backoff," IEEE/ACM Transactions on Networking, vol. 26, no. 1, pp. 451-464, Feb 2018.

[12] - "The access delay of Aloha with exponential back-off strategies," in 2016 Mediterranean Ad Hoc Networking Workshop (Med-Hoc-Net), June 2016, pp. 1-6.

[13] L. Barletta and F. Borgonovo, "The stability of exponential backoff protocols for slotted-Aloha with saturated queues," in IEEE Information Theory Workshop (ITW), Nov. 2017, pp. 1-5.

[14] J. D. C. Little, "A proof for the queuing formula: $\mathrm{L}=\lambda \mathrm{W}$," Operations Research, vol. 9, no. 3, pp. 383-387, 1961.

[15] A. Sen and N. Balakrishnan, "Convolution of geometrics and a reliability problem," Statistics \& Probability Letters, vol. 43, no. 4, pp. 421 - 426, 1999. [Online]. Available: http://www.sciencedirect.com/science/article/pii/S0167715298002843 